\title{
Futilidade Terapêutica e Insuficiência Respiratória: Realização de um Estudo de Coorte Prospectiva*
}

\author{
Medical Futility and Respiratory Failure: A Prospective Cohort Study
}

Cristiano Corrêa Batista1, Maurício Alberto Goldbaum Júnior², Fernanda Sztiler2, José Roberto Goldim³, Carlos Cezar Fritscher ${ }^{4}$

\section{RESUMO}

JUSTIFICATIVA E OBJETIVOS: A necessidade de reformular as metas dos cuidados intensivos, partindo muitas vezes da cura para o conforto, torna-se necessário nos dias atuais. O médico intensivista, freqüentemente está diante da decisão de suspender ou não oferecer determinado tratamento, apesar de ele estar disponível. O objetivo deste estudo foi estimar o risco evolutivo de probabilidade de morte individual para pacientes internados com insuficiência respiratória na unidade de terapia intensiva (UTI), identificar quais os tratamentos mantenedores da vida foram administrados, o tempo de internação e o desfecho, comparar o desfecho "morte" em relação aos modelos UNICAMP II e APACHE II, bem como verificar se os tratamentos mantenedores da vida podem ser limitados ou suspensos.

MÉTODO: Trata-se de um estudo de coorte prospectiva, observacional envolvendo 150 pacientes com insuficiência respiratória internados na unidade de tratamento intensivo. A análise estatística foi realizada por meio dos Modelos Lineares Generalizados.

RESULTADOS: Idade, sexo, raça ou morbidade não

1. Médico Intensivista, Bioeticista, Professor da UCPel, PhD

2. Acadêmico de Medicina da PUCRS

3.Biólogo, Bioeticista, Professor da UFRGS/PUCRS, PhD

4. Médico Pneumologista, Professor da PUCRS, PhD

*Recebido do Hospital Universitário São Lucas da Pontifícia Universidade Católica do Rio Grande do Sul (PUCRS), Porto Alegre, RS

Apresentado em 29 de dezembro de 2006

Aceito para publicação em 17 de abril de 2007

Endereço para correspondência:

Dr. Cristiano Corrêa Batista

Rua Três de Maio, 700

96010-770 Pelotas, RS

E-mail: cbatistasul@hotmail.com

(C)Associação de Medicina Intensiva Brasileira, 2007 mostrou estatística significativa para predizer o desfecho. Essa predição foi mais bem averiguada por meio da evolução do índice prognóstico individual nos primeiros sete dias de internação na UTI. A piora do prognóstico em $10 \%$ para pacientes com risco inicial entre $70 \%$ e $80 \%$, utilizando o modelo UNICAMP II, mostrou especificidade de $97,4 \%$ a $98,6 \%$.

CONCLUSÕES: A evolução prognóstica dos pacientes, nos primeiros sete dias de internação na UTI, é de grande auxílio do ponto de vista objetivo para a tomada de decisões éticas em torno da não-oferta de novos tratamentos mantenedores da vida.

Unitermos: Insuficiência Respiratória, Futilidade Terapêutica, UTI

\section{SUMMARY}

BACKGROUND AND OBJECTIVES: Currently, the reformulation of intensive care goals, often shifting from the search for a cure to offering comfort, has become more and more necessary. The intensivist is frequently confronted with the decision to suspend or not offer a specific therapy, despite its availability. The objective of this study was to estimate the developing risk of probability of death for individual ICU patients with respiratory failure, identify which life-sustaining therapies were administered, time of internment and outcome. Compare the death outcome in relation to UNICAMP II and APACHE II models, as well as verify if the life-sustaining therapies may be limited or suspended.

METHODS: It is the observational, prospective cohort study of 150 patients with respiratory failure confined to the intensive care unit. Statistical analysis was carried out using Generalized Linear Models.

RESULTS: Age, sex, race or morbidity did not reveal statistical significance in predicting outcome. This prediction was confirmed more accurately by means of changes in the individual prognostic index of death probability during the first seven days of ICU internment. 
A $10 \%$ worsening prognosis in patients who presented initial death risk of $70 \%$ to $80 \%$, utilizing the UNICAMP II Model, showed a specificity of $97.4 \%-98.6 \%$.

CONCLUSIONS: Prognostic changes in patients during the first seven days of ICU internment are of great aid, from an objective point of view, for ethical decision-making in relation to not-offering new life-sustaining therapies.

Key Words: ICU, Medical Futility, Respiratory Failure

\section{INTRODUÇÃO}

Quando um tratamento fútil, no sentido de medicamento inapropriado ou não-benéfico, pode ser recusado aos pacientes ou suspenso? Somente quando os pacientes recusarem aceitá-lo? Ou ele pode ser também suspenso ou não-ofertado quando profissionais tiverem a consciência de sua inutilidade?

Tem-se considerado tratamentos fúteis, quando apresentam menos de $1 \%$ de probabilidade de sucesso ou se, provavelmente, nunca terminará com a dependência dos cuidados intensivos ${ }^{1}$. A habilidade em determinar, de forma acurada, o prognóstico, tanto no momento do ingresso na UTI quanto na resposta subseqüente ao tratamento, melhoraria, de forma consistente, a prática médica no ambiente de cuidados intensivos. A avaliação do risco quantitativo não somente realçaria a prática clínica, como também contribuiria para avaliar a qualidade dos cuidados dispensados aos pacientes e permitiria justa distribuição dos recursos disponíveis ${ }^{2}$.

A Sociedade Americana de Medicina Intensiva (The Society of Critical Care Medicine) tem apoiado as decisões de limitação de tratamento em pacientes terminais. Ela refere que a habilidade de um sistema de predição, em discernir quais pacientes irão morrer oferece a possibilidade de alocar, de forma justa, os recursos na UTI e assim promover o princípio da justiça distributiva. $\mathrm{O}$ mecanismo para alcançar tal percepção deveria apresentar alta especificidade na determinação de quem irá morrer, quando poucos pacientes sobreviveriam se o tratamento fosse continuado ou suspenso ${ }^{3}$.

O objetivo deste estudo foi avaliar o risco evolutivo de probabilidade de morte individual para pacientes internados com insuficiência respiratória na UTI, utilizando o modelo UNICAMP II ${ }^{4}$; Identificar quando os tratamentos mantenedores da vida foram empregados, o tempo de internação na UTI e o desfecho; comparar o grau de risco evolutivo de probabilidade morte individual e o desfecho morte em relação aos modelos UNICAMP II e APACHE II5; verificar se os tratamentos mantenedoras da vida poderiam ser limitados ou suspensos, basea- do em um sistema evolutivo de probabilidade de óbito utilizando o modelo UNICAMP II.

\section{MÉTODO}

Trata-se de um estudo de coorte prospectiva, contemporânea, observacional, desenvolvido na unidade geral de tratamento intensivo (UTI) com 14 leitos, do Hospital Universitário São Lucas, da Pontifícia Universidade Católica do Rio Grande do Sul (PUCRS), na cidade de Porto Alegre, estado do Rio Grande do Sul, Brasil. O estudo foi aprovado pelo Comitê de Ética em Pesquisa da PUCRS. O período de coleta de dados foi de $1^{\circ}$ de setembro de 2002 a 31 de dezembro de 2003. Os critérios de inclusão foram: pacientes internados na UTI no período do estudo, motivados por quadro de insuficiência respiratória aguda ou crônica agudizada. Os pacientes deveriam ser proveniente da unidade de emergência do Hospital São Lucas, dos leitos de internação hospitalar e da sala de recuperação pós-anestésica. Os critérios de exclusão foram: os pacientes oriundos do centro cirúrgico para recuperação pós-operatória previamente programada, os pacientes internados na UTI transferidos de outro hospital, os pacientes com complicações durante o procedimento cirúrgico necessitando de tratamento intensivo imediato, os pacientes que desenvolveram insuficiência respiratória após estarem internados na UTI por mais de 24 horas e os pacientes nos quais não foi possível a obtenção do consentimento informado.

Definiu-se como critério de insuficiência respiratória o fato de os pacientes apresentarem, na gasometria arterial, pressão arterial de oxigênio menor ou igual a 50 $\mathrm{mmHg}\left(\mathrm{PaO}_{2} \leq 50 \mathrm{mmHg}\right)$ ou pressão arterial de dióxido de carbono maior ou igual a $50 \mathrm{mmHg}\left(\mathrm{PaCO}_{2} \geq \mathrm{mmHg}\right)$, independentemente da causa básica que a motivou. Todos os pacientes internados incluídos no estudo foram acompanhados, desde o momento do ingresso até o desfecho: alta para a unidade ou óbito. O protocolo de acompanhamento consistiu de um instrumento de registro de dados obtido, diariamente, a partir dos prontuários dos pacientes. Foi estimado o risco provável de óbito, por meio do índice prognóstico UNICAMP II, em um sistema evolutivo de sete dias, sendo registradas as estimativas de óbito no $1^{\circ}$ dia de internação e, subseqüentemente, no $3^{\circ}, 5^{\circ}$ e $7^{\circ}$ dia. Além disso, foram anotados o tempo de internação na unidade (dias), o momento do emprego dos tratamentos e, posteriormente, verificado o desfecho.

Utilizou-se igualmente o sistema de estimação de risco de morte hospitalar APACHE II como comparativo ao 
sistema UNICAMP II. Foram assumidos como tratamentos mantenedores da vida: a ventilação mecânica invasiva, a hemodiálise, a nutrição parenteral total, as transfusões de sangue e derivados e os fármacos vasoativos (dopamina, dobutamina e noradrenalina). Definiu-se como benefício dos tratamentos, a sobrevida do paciente, seguida de alta da UTI para os leitos de tratamento hospitalar e, como malefício, o óbito. Foi considerada como possível terapia fútil, a oferta de tratamentos mantenedores da vida para aqueles pacientes com aumento progressivo do risco estimado de óbito nos primeiros sete dias de internação na UTI e ou risco estimado de óbito $\geq 90 \%$.

\section{Análise Estatística}

Inicialmente, realizou-se uma análise descritiva dos dados e, posteriormente, foram estruturadas categorias de morbidade, idade e tempo do emprego dos tratamentos mantenedores da vida. A seguir, foi verificada a distribuição da amostra, de acordo com o tempo médio de internação na unidade, pelos testes $t$ de Student para as variáveis dicotômicas e Análise de Variância (ANOVA) para as variáveis politômicas. A distribuição da amostra de acordo com o desfecho, bem como a relação das suas intervenções, foram realizadas por meio do teste Qui-quadrado. Posteriormente, utilizouse a Análise de Variância para verificar as médias das estimativas de risco provável de óbito, pelo sistema UNICAMP II, no $1^{\circ}, 3^{\circ}, 5^{\circ}$ e $7^{\circ}$ dias de internação e para compará-las com o desfecho.

Verificou-se, também, a sensibilidade, a especificidade, os valores preditivos positivo e negativo da estimação de risco UNICAMP II em relação ao desfecho, quando os pacientes apresentassem risco estimado de óbito maior ou igual a 90\%. Para apurar a intensidade e a direção da associação linear entre os índices prognósticos UNICAMP II e APACHE II foram realizados o teste de correlação de Pearson. As estimativas de risco de morte individual foram avaliadas usando um valor $\pi$ (pi) a partir dos índices UNICAMP II e APACHE II no $1^{\circ}$ dia de internação e, a seguir, verificados os ajustes estatísticos pelos valores de Deviance e Akaike Information Criteria (AIC). A mesma análise foi realizada para a estimativa de morte individual no $7^{\circ}$ dia. Por considerar-se que a evolução do índice prognóstico durante os primeiros sete dias de internação na UTI contém informações importantes e complementares à medida prognóstica no primeiro dia, criou-se, então, nova variável definida como variável de evolução prognóstica que é a diferença do $7^{\circ}$ dia em relação ao primeiro dia. Para os pacientes com permanência por mais de sete dias na UTI, foi realizada uma análise pelos Modelos Lineares Generalizados $(\mathrm{MLG})^{6,7}$, binomial, logístico, para previsão de risco de mortalidade na UTI $\pi$ em função de algumas variáveis explicativas tanto para o índice APACHE II quanto para o UNICAMP II tais como: a) o índice prognóstico medido no primeiro dia; b) a evolução prognóstica nos sete primeiros dias; c) o sexo; d) a cor; e) a categoria de morbidade. Para verificar qual a melhor combinação de variáveis explicativas para cada índice prognóstico e para identificar qual dos dois índices é o mais eficiente, foram feitas comparações entre os vários modelos pelos valores de Deviance e AIC.

A estimativa do risco de morte individual $\pi$ foi calculada da seguinte maneira: Yi é uma variável aleatória binária que identifica o desfecho para o paciente e segue distribuição binomial com probabilidade de óbito $\pi \mathrm{i}$. Estabeleceu-se uma relação linear entre a transformação logística com variáveis explicativas, onde $\eta i$ $(n u)=\log (\pi \mathrm{i} /(1-\pi \mathrm{i}))=\mathrm{B} 0+\mathrm{B} 1 \mathrm{x}$ índice prognóstico $1^{\circ}$ dia + B2 x índice de evolução prognóstica 7/1. Então $\pi \mathrm{i}=\exp (\eta \mathrm{i}) /(1+\exp (\eta \mathrm{i}))$. Com base nesse modelo, foram calculados os valores de $\pi$ para cada paciente que permaneceu na UTI por mais de sete dias, com o objetivo de avaliar eventuais diferenças nos valores de $\pi$ para diferentes categorias de pacientes. A fim de verificar qual seria o melhor ajuste estatístico do índice prognóstico evolutivo $\pi$, realizou-se comparações pelos valores de AIC para Modelos Lineares Generalizados que utilizam duas variáveis explicativas: o índice prognóstico no dia da internação e a evolução no índice no $3^{\circ}, 5^{\circ}$ e $7^{\circ}$ dia.

Para verificar o comportamento da curva de características operacionais (Curva ROC) para ambos os índices prognósticos, a partir dos dados do estudo, constatou-se ser necessário um banco de dados muito maior. Então, como os dados obtidos no estudo representam a realidade, calculou-se, inicialmente, um banco de dados virtual com 5.000 pacientes a partir do banco de dados obtido durante o estudo. Para realizar essa tarefa, primeiramente fixou-se um índice prognóstico e um dia de internação $\left(1^{\circ}, 3^{\circ}, 5^{\circ}\right.$ e $7^{\circ}$ ). Depois, foram estimados os parâmetros do modelo de regressão logística para $\pi$, utilizando todos os pacientes disponíveis por dia de internação. Então, foram gerados 5.000 pacientes com valores do índice distribuídos ao acaso entre zero e 100 e realizou-se o cálculo das estimativas de $\pi$ utilizando o modelo ajustado aos dados. Fixou-se um valor do índice prognóstico (D0) e foi classificado como 
possível óbito todo paciente cujo índice superasse D0 (D0 (\%) = 1, 5, 10, 30, 40, $50,60,70,80,90,95$, 99). Associou-se óbito a cada um dos 5.000 pacientes de acordo com as probabilidades $\pi$ preditas pelo modelo que foi ajustado aos dados reais. Então foi calculada a porcentagem de possíveis óbitos entre todos os óbitos (sensibilidade) e a porcentagem de possíveis óbitos entre todas as altas (1 - especificidade) para cada valor de D0.

Por fim criou-se um banco de dados simulado de 10.000 pacientes para os quais foi estimado o risco de óbito $\pi$ utilizando os Modelos Lineares Generalizados que, além do índice prognóstico no $1^{\circ} \mathrm{dia}$, incluíam a variável de evolução prognóstica. Foram estipulados níveis de evolução até o $7^{\circ}$ dia, como possível óbito (índice de evolução prognóstica) de - 20\%, -10\% e $+10 \%$. Os valores positivos significaram piora prognóstica do paciente e valores negativos, melhora prognóstica e foi verificada uma nova curva ROC para estimativa do possível óbito para cada um desses índices de evolução prognóstica.

\section{RESULTADOS}

Durante o período de coleta de dados, foram internados na UTI 166 pacientes com quadro de insuficiência respiratória aguda e que preenchiam os critérios de inclusão no estudo. Desses, 16 pacientes foram excluídos por não ter sido possível a obtenção do consentimento informado. Os 150 pacientes restantes foram acompanhados desde o dia de sua internação até o desfecho. Os dados demográficos estão apresentados na tabela 1.

Tabela 1 - Variáveis Demográficas dos Pacientes Estudados

\begin{tabular}{lc}
\hline Variáveis & $\mathrm{N}(\%)$ \\
\hline Sexo & \\
Masculino & $95(63)$ \\
Feminino & $55(37)$ \\
Idade (anos) & \\
$\quad$ Mínima & 16 \\
Máxima & 95 \\
Média \pm DP & $56,89 \pm 19,22$ \\
Morbidade básica (doença) & \\
Neurológica & $16(10,7)$ \\
Neoplásica & $29(19,3)$ \\
Infecciosa & $29(19,3)$ \\
Cardiopulmonar & $35(23,3)$ \\
Renal & $7(4,7)$ \\
Gastrintestinal & $4(2,7)$ \\
Cirúrgica e trauma & $18(12)$ \\
Endócrina e outras & $12(8)$ \\
\hline
\end{tabular}

Nesse estudo idade, sexo e raça não mostraram diferença estatística significativa entre grupos no referente ao desfecho morte. A morbidade inicialmente foi classificada em 3 categorias: 1) apresentou doenças neurológicas, cirúrgicas e traumas; 2) abrangeu doenças cardíacas, pulmonares, gastrintestinais, renais e infecciosas; 3) abarcou doenças neoplásicas, endócrinas e outras. Não houve diferença estatística significativa entre os grupos $(p=0,438)$.

Em relação ao tempo médio, em dias, de internação na UTI, não houve diferença estatística significativa para sexo, raça e idade, mas sim, para morbidade. Os pacientes agrupados na categoria 1 de morbidade permaneceram por mais tempo internados na UTI $(22,15$ dias) do que os pacientes das outras categorias $(p=$ $0,002)$.

Entre os tratamentos mantenedores da vida, a ventilação mecânica invasiva foi a mais utilizada, seguido dos fármacos vasoativos, transfusão de sangue e derivados. Entre os fármacos vasoativos, a noradrenalina foi a mais comumente usada e a dopamina, a menos utilizada. Entre os pacientes submetidos à ventilação mecânica $(n=136), 133$ a iniciaram antes dos $7^{\circ}$ dia de internação e três, após. Dentre aqueles pacientes que iniciaram antes dos $7^{\circ}$ dia $76(57,1 \%)$ tiveram alta e $57(42,9 \%)$ foram a óbito. Todos os pacientes que iniciaram ventilação mecânica após $\circ 7^{\circ}$ dia foram a óbito. Entre os pacientes que não receberam ventilação mecânica $(n=14), 13(92,9 \%)$ tiveram alta e um $(7,1 \%)$ foi a óbito. Houve diferença estatística significativa entre os grupos em relação ao desfecho $(p=0,004)$. Não houve diferença estatística entre os grupos em relação ao tempo médio de ventilação ( $p$ $=0,245$ ). A dopamina foi empregada em apenas um paciente o qual foi a óbito e, portanto, essa variável não foi incluída na análise estatística. A dobutamina foi utilizada em 26 pacientes: 21 iniciaram antes do $7^{\circ}$ dia e cinco após. Entre aqueles que receberam dobutamina antes do $7^{\circ}$ dia, $12(57,1 \%)$ tiveram alta e nove $(42,9 \%)$ foram a óbito e, entre os outros que iniciaram dobutamina depois do $7^{\circ}$ dia, um (20\%) teve alta e quatro $(80 \%)$ foram a óbito. Dos 124 pacientes que não receberam dobutamina, $76(61,3 \%)$ tiveram alta e $48(38,7 \%)$ foram a óbito. Não houve diferença estatística significativa entre os grupos tanto em relação ao desfecho $(p=0,179)$ quanto ao tempo médio de uso do fármaco $(p=0,532)$. A noradrenalina foi administrada a 90 pacientes: 84 iniciaram antes do $7^{\circ}$ dia e seis após. Dos pacientes que iniciaram noradrenalina antes do $7^{\circ}$ dia, $39(46,4 \%)$ tiveram alta e $45(53,6 \%)$ 
foram a óbito. Entre aqueles que receberam noradrenalina depois do $7^{\circ}$ dia, um $(16,7 \%)$ teve alta e cinco $(83,3 \%)$ foram a óbito. Para os 60 pacientes que não utilizaram noradrenalina, 49 pacientes $(81,7 \%)$ tiveram alta e $11(18,3 \%)$ foram a óbito. Houve diferença estatística significativa em relação ao desfecho $(p<$ 0,001), mas não houve diferença estatística significativa em relação ao tempo médio do uso do fármaco $(p$ $=0,601)$. Foi administrada transfusão de sangue e derivados para 54 pacientes: sendo que 43 receberam transfusão antes do $7^{\circ}$ dia e 11 após. Entre os pacientes que a receberam antes do $7^{\circ}$ dia, $13(30,2 \%)$ tiveram alta e $30(69,8 \%)$ foram a óbito. Entre aqueles que a receberam depois do $7^{\circ}$ dia, cinco $(45,5 \%)$ tiveram alta e seis $(54,5 \%)$ foram a óbito. Dos pacientes que não receberam transfusão de sangue e derivados, 71 (74\%) tiveram alta e 25 (26\%) foram a óbito. Houve diferença estatística significativa entre os grupos em relação ao desfecho $(p<0,001)$, mas não quanto ao número de transfusões $(p=0,091)$. A hemodiálise foi ofertada para 13 pacientes: oito a iniciaram antes do $7^{\circ}$ dia e cinco após. Para aqueles com início antes do $7^{\circ}$ dia, dois (25\%) tiveram alta e seis $(75 \%)$ foram a óbito. Para os pacientes que iniciaram hemodiálise após $07^{\circ}$ dia constatou-se óbito para todos. Entre os pacientes que não receberam hemodiálise, 87 $(63,5 \%)$ tiveram alta e $50(36,5 \%)$ foram a óbito. Houve diferença estatística significativa entre os grupos com relação ao desfecho $(p=0,002)$ mas não quanto ao tempo médio de uso da hemodiálise $(p=0,309)$. A nutrição parenteral total (NPT) foi ofertada para 13 pacientes: 12 iniciaram antes do $7^{\circ}$ dia e um após. Entre os pacientes que receberam NPT antes do $7^{\circ}$ dia, quatro $(33,3 \%)$ tiveram alta e oito $(66,7 \%)$ foram a óbito assim como o paciente que recebeu NPT após o $7^{\circ}$ dia. Entre os pacientes que não receberam NPT, $85(62 \%)$ tiveram alta e 52 (38\%) foram a óbito. Não houve diferença estatística significativa entre os grupos tanto em relação ao desfecho $(p=0,073)$ quanto ao tempo médio de uso da NPT ( $p=0,752)$. Os escores de risco medidos, pelo sistema UNICAMP II, entre os pacientes com alta ou óbito no $1^{\circ}, 3^{\circ}, 5^{\circ}$ e $7^{\circ}$ dia de internação mostraram diferença estatística significativa entre os grupos em todos os momentos. Os pacientes que foram a óbito, em média apresentaram risco provável de óbito maior do que aqueles com alta ( $p<0,001$ ) (Figura 1). Ao longo dos primeiros sete dias de internação na UTI, 17 pacientes apresentaram risco provável de óbito $\geq 90 \%$. Desses, três $(17,6 \%)$ tiveram alta e $14(82,4 \%)$ foram a óbito. A sensibili- dade verificada para pacientes com risco de óbito $\geq$ 90\%, medido pelo sistema UNICAMP II, foi de $23 \%$ e a especificidade, de $97 \%$; o valor preditivo positivo de $82,4 \%$ e o valor preditivo negativo de $64,7 \%$. A comparação inicial entre os sistemas UNICAMP II e APACHE II quanto à intensidade e à direção da associação linear mostrou existir forte correlação entre ambas as medidas em todos os tempos de aferição $\left(1^{\circ}\right.$ dia $r=0,843 ; 3^{\circ}$ dia $r=0,839 ; 5^{\circ}$ dia $r=0,855 ; 7^{\circ}$ dia $r=0,854),(p<0,001)$.

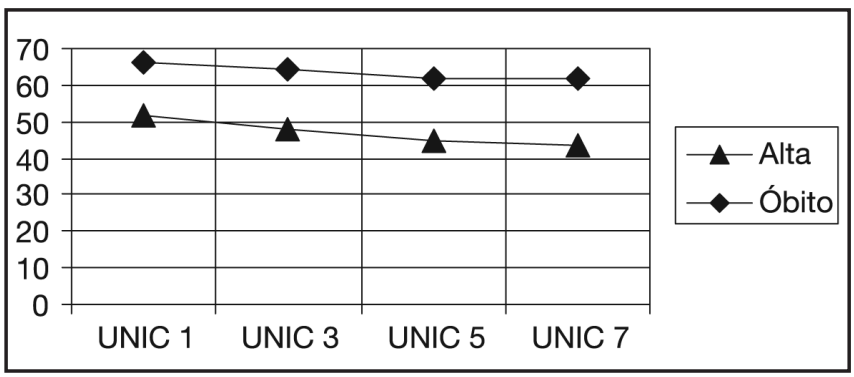

Figura 1 - Médias de UNICAMP II de Acordo com o Desfecho UNIC $1=$ UNICAMPII no $1^{\circ}$ dia; UNIC $3=$ UNICAMP II no $3^{\circ}$ dia; UNIC $5=$ UNICAMP no $5^{\circ}$ dia; UNIC 7 = UNICAMP $\|$ no $7^{\circ}$ dia. $p<0,001$

As medidas de risco de morte individual "pi" $(\pi)$, na UTI, estimadas a partir dos índices prognósticos APACHE II e UNICAMP II no $1^{\circ}$ dia e ajustadas pelos níveis descritivos para valores de Deviance e AIC mostraram que as qualidades dos ajustes estatísticos foram semelhantes para os dois modelos e ambos estatisticamente significativos, com discreta vantagem para o APACHE II. Entre os 150 pacientes constituintes do banco de dados original, 112 pacientes permaneceram internados na UTI por mais de sete dias. As exclusões foram devidas a 20 pacientes com alta e 18 pacientes com óbito. Existiu uma marcante diferença entre os grupos. Para os pacientes que foram a óbito, nenhum valor de UNICAMP II foi inferior a $47,10 \%$, enquanto para aqueles que tiveram alta, $50 \%$ dos índices observados foram inferiores a $45,40 \%$. Verificouse uma dispersão levemente superior para o índice APACHE II. Entre os 112 pacientes internados por 7 dias ou mais, 69 (62\%) resultaram em alta e 43 (38\%) em óbito. A partir da criação da variável de evolução prognóstica, verificou-se, que entre os pacientes que tiveram alta, a evolução dos índices prognósticos foi bem mais acentuada do que para aqueles que resultaram em óbito. Ao verificar-se a eficiência dos índices prognósticos, a partir das comparações entre os valores de Deviance e AIC, constatou-se que ambos 
os modelos, tanto para o sistema APACHE II quanto para o UNICAMP II, que utilizaram a combinação de variáveis prognósticas, $1^{\circ}$ dia e evolução do $7^{\circ} \mathrm{em}$ relação ao $1^{\circ}$ dia, foram os modelos com os menores valores de AIC. O acréscimo das outras variáveis não compensou o custo representado pelo aumento na complexidade do modelo. Constatou-se que tanto o modelo com o sistema APACHE II (Deviance = $0,106)$ quanto o modelo com o sistema UNICAMP ॥ (Deviance $=0,073$ ) são aceitáveis. Ao verificar-se qual teria sido a melhor alternativa de evolução do índice prognóstico entre $\circ 1^{\circ}$ dia e $\circ 7^{\circ}$ dia, baseados na comparação entre as diferenças do $3^{\circ}, 5^{\circ}$ e $7^{\circ}$ dia pelos menores valores de $\mathrm{AIC}$, constatou-se que as melhores qualidades de ajustes foram representadas pela evolução do $7^{\circ}$ dia em relação ao $1^{\circ}$ dia, para o UNICAMP II (AIC = 137,13) e para o APACHE II (AIC $=133,71)$. Com base nesse modelo de probabilidade de morte individual $\pi$, foram calculadas as estimativas de óbito para cada um dos 112 pacientes internados na UTI por sete ou mais dias e comparadas com as variáveis que foram consideradas como tratamentos mantenedores da vida. Os resultados estão apresentados na tabela 2. As curvas de características operacionais (Curva $\mathrm{ROC}$ ) construídas a partir de um banco de dados com 10000 pacientes, para o sistema UNICAMP II a partir da classificação como possível óbito, em função da variável de evolução prognóstica, de $-20 \%,-10 \%$ e $10 \%$ são mostradas na figura 2.

Tabela 2 - Probabilidade de Morte Individual $\pi$ para os 112 Pacientes que Permaneceram Internados na UTI por 7 ou mais Dias

\begin{tabular}{|c|c|c|c|c|c|c|c|}
\hline Variáveis & Mínimo & Mediana & Média & Máximo & $\mathrm{DP}$ & $\mathrm{n}$ & Valor $\mathrm{p}$ \\
\hline \multicolumn{8}{|l|}{ Desfecho } \\
\hline Alta & 0,06 & 0,28 & 0,32 & 0,72 & 0,17 & 69 & \multirow[t]{2}{*}{$<0,001$} \\
\hline Óbito & 0,17 & 0,46 & 0,47 & 0,79 & 0,18 & 43 & \\
\hline \multicolumn{8}{|l|}{ VMI } \\
\hline Antes & 0,06 & 0,38 & 0,39 & 0,79 & 0,19 & 103 & \multirow[t]{3}{*}{0,024} \\
\hline Depois & 0,22 & 0,26 & 0,31 & 0,46 & 0,13 & 3 & \\
\hline Não usou & 0,13 & 0,15 & 0,18 & 0,29 & 0,06 & 6 & \\
\hline \multicolumn{8}{|l|}{ Dobutamina } \\
\hline Antes & 0,07 & 0,48 & 0,44 & 0,64 & 0,16 & 18 & \multirow[t]{3}{*}{0,18} \\
\hline Depois & 0,22 & 0,42 & 0,46 & 0,68 & 0,17 & 5 & \\
\hline Não usou & 0,06 & 0,34 & 0,36 & 0,79 & 0,19 & 89 & \\
\hline \multicolumn{8}{|l|}{ Noradrenalina } \\
\hline Antes & 0,06 & 0,42 & 0,43 & 0,79 & 0,18 & 64 & \multirow[t]{3}{*}{0,003} \\
\hline Depois & 0,16 & 0,24 & 0,28 & 0,46 & 0,12 & 6 & \\
\hline Não usou & 0,07 & 0,26 & 0,32 & 0,75 & 0,18 & 42 & \\
\hline \multicolumn{8}{|l|}{ NPT } \\
\hline Antes & 0,29 & 0,48 & 0,51 & 0,75 & 0,16 & 8 & \multirow[t]{3}{*}{0,003} \\
\hline Depois & 0,78 & 0,78 & 0,78 & 0,78 & - & 1 & \\
\hline Não usou & 0,06 & 0,35 & 0,37 & 0,79 & 0,18 & 103 & \\
\hline \multicolumn{8}{|l|}{ Hemodiálise } \\
\hline Antes & 0,27 & 0,65 & 0,57 & 0,72 & 0,16 & 7 & \multirow[t]{3}{*}{0,01} \\
\hline Depois & 0,28 & 0,42 & 0,46 & 0,64 & 0,14 & 5 & \\
\hline Não usou & 0,06 & 0,34 & 0,36 & 0,79 & 0,18 & 100 & \\
\hline \multicolumn{8}{|l|}{ Transfusão } \\
\hline Antes & 0,14 & 0,47 & 0,47 & 0,78 & 0,18 & 31 & \multirow[t]{3}{*}{0,001} \\
\hline Depois & 0,28 & 0,42 & 0,44 & 0,75 & 0,13 & 11 & \\
\hline Não usou & 0,06 & 0,28 & 0,33 & 0,79 & 0,18 & 70 & \\
\hline \multicolumn{8}{|l|}{ Morbidade } \\
\hline Categoria 1 & 0,09 & 0,36 & 0,39 & 0,79 & 0,20 & 50 & \multirow[t]{3}{*}{0,46} \\
\hline Categoria 2 & 0,16 & 0,44 & 0,43 & 0,67 & 0,17 & 16 & \\
\hline Categoria 3 & 0,06 & 0,35 & 0,36 & 0,73 & 0,18 & 46 & \\
\hline
\end{tabular}

VMI = ventilação mecânica invasiva; NPT = nutrição parenteral total; Categoria 1 = neurológico/sepse /cirurgia /trauma; Categoria 2 = neoplasia; Categoria 3 = cardiopulmonar/renal/gastrintestinal/endocrinológica e outras. 


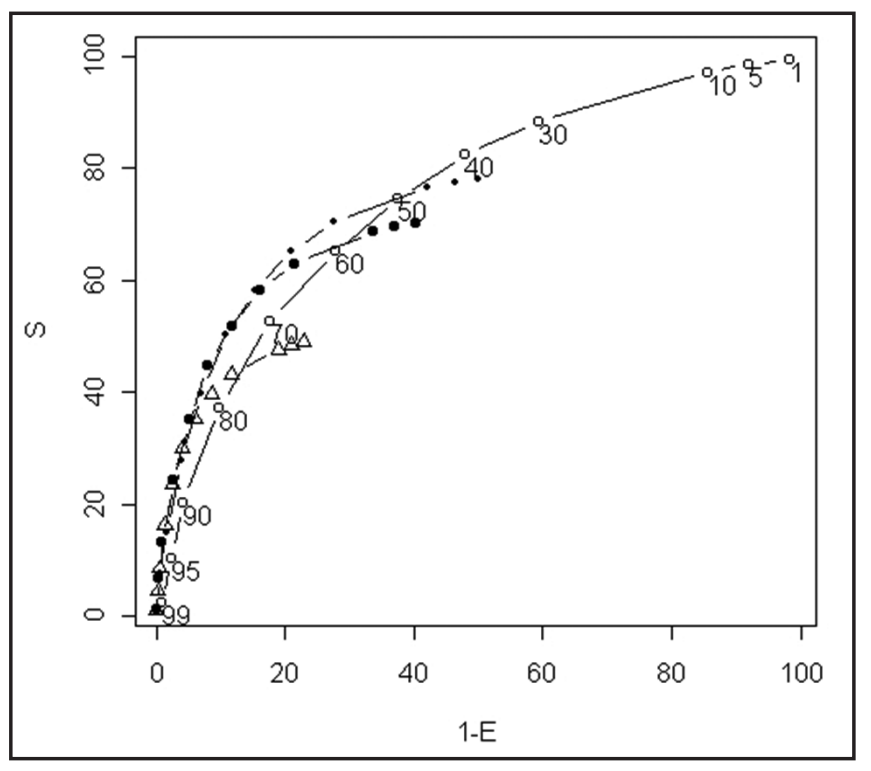

Figura 2 - Curvas ROC Obtidas para Modelos que ajustam Probabilidade de Óbito em Função do Índice UNICAMP II

Os círculos vazados representam os valores do índice prognóstico utilizado como limite mínimo para classificar o paciente como possível óbito. Os círculos pretos representam as curvas ROC para modelos que ajustam probabilidade de óbito em função do índice prognóstico UNICAMP II no $1^{\circ}$ dia e a diferença em relação ao $7^{\circ}$ dia. Os valores do índice utilizado como limite para classificar o paciente como possível óbito foram $-10 \%$ em relação à diferença do $7^{\circ}$ para o $1^{\circ}$ dia. Os pontos pretos representam o valor limite da diferença entre $7^{\circ}$ e $1^{\circ}$ dia foi $-20 \%$ e os triângulos vazados representam a diferença de $+10 \%$. $\mathrm{S}=$ sensibilidade; $1-\mathrm{E}=1-$ especificidade.

\section{DISCUSSÃO}

A avaliação prognóstica utilizando o modelo preditivo UNICAMP II, mediante um sistema evolutivo, para pacientes em estado crítico com insuficiência respiratória é de grande auxílio para o médico intensivista na tomada de decisão da não-oferta de novos tratamentos mantenedores da vida.

A partir deste estudo, pode-se constatar que variáveis demográficas tais como idade, sexo, raça e morbidade não mostraram significância estatística para predizer o desfecho. Entendemos, portanto, que essas variáveis isoladas não deveriam ser a base dos debates em torno da futilidade terapêutica. Elas poderiam, talvez, de algum modo, contribuir para os debates sob o ponto de vista subjetivo, mas certamente não deveriam ser consideradas em primeiro plano.

Uma variedade de fatores têm sido investigados para predizer resultados e fatores demográficos como idade e morbidade são, muitas vezes, incluídos ${ }^{8}$. Apesar da identificação de grupos particulares de pacientes com alta mortalidade hospitalar, não se pode sugerir que, a partir do diagnóstico de uma doença crônica ou aguda fatal, raça ou idade, haveria impedimento do uso efeti- vo do cuidados intensivos. Estudos também enfocam a idade, grupo diagnóstico e gravidade da doença como critérios de triagem para a recusa de internação dos pacientes em unidades de cuidados intensivos sob a argumentação lógica e prudente do princípio da justa alocação de recursos escassos ${ }^{9}$.

Esses critérios procuram enfatizar suas bases na mortalidade hospitalar. No entanto, para o intensivista, a questão é ainda mais complexa. Uma vez o paciente sendo admitido na unidade, mesmo existindo conflitos em relação aos critérios de admissibilidade, a partir de então, os principais dilemas passam a girar em torno da mortalidade na UTI e não a hospitalar, bem como no prolongamento individual do processo de morrer, o que configuraria inadequada prática médica e o desrespeito ao princípio bioético da não-maleficência. Nesse estudo, identificou-se que a morbidade interferiu, de forma significativa, no prolongamento do tempo de internação na unidade mas não no aumento da mortalidade na UTI. Sendo assim, sob a sua visão, tais critérios, objetivamente, ficariam em segundo plano na tomada de decisão quanto alocar recursos. O princípio da justiça seria contemplado na medida em que passa a existir a consciência de o aumento dos custos ser devido à maior permanência do paciente na unidade, ocasionada pela gravidade da doença, e essa permanência não implicar no aumento de óbitos na unidade. A não-oferta de tratamentos efetivos, cujo alicerce principal são variáveis demográficas, conforme esses resultados, não se justifica.

Entre os tratamentos administrados, verificou-se terem sido a ventilação mecânica invasiva e os fármacos vasoativos mais freqüentemente utilizados, seguidas pela transfusão de sangue e derivados, hemodiálise e nutrição parenteral total. Na maioria das vezes, os tratamentos foram iniciados antes do $7^{\circ}$ dia de internação na UTI, demonstrando ser esse período, geralmente, o de maior complexidade e instabilidade do quadro clínico dos pacientes. Assim, justifica-se a tentativa incessante, durante esse período, de proporcionar a probabilidade de recuperação aos pacientes críticos por meio dos recursos técnicos, independentemente do seu grau de complexidade, desenvolvidos pela Medicina.

A constatação da maior freqüência no uso da ventilação mecânica e dos fármacos vasoativos como tratamentos efetivos reflete a realidade do seu uso em UTI e seria lógico esperar que pacientes admitidos devido ao quadro de insuficiência respiratória fossem mais freqüentemente submetidos a eles. Pacientes em 
insuficiência respiratória, muitas vezes, apresentam alterações hemodinâmicas associadas ao quadro ou corroboradas pelo próprio uso do suporte ventilatório invasivo. Interessante foi verificar que o tempo de uso, em dias, desses tratamentos, bem como o número de transfusões não se mostrou estatisticamente significativo para o desfecho. Isto demonstra que o tempo de seu uso não implicou no aumento da mortalidade na UTI e, portanto, também não seria plausível a justificativa de discutir futilidade terapêutica em torno do tempo prolongado da oferta desses tratamentos. Pareceu-nos mais razoável, a partir dos resultados desse estudo, abrir discussões em torno de quando esses tratamentos foram iniciados. Verificou-se terem ido a óbito todos os pacientes que iniciaram ventilação mecânica $(n=3)$ ou hemodiálise $(n=5)$ após o $7^{\circ}$ dia. Entre aqueles que iniciaram dobutamina após o $7^{\circ}$ dia, $80 \%$ foram a óbito e entre os que iniciaram noradrenalina, $83 \%$ também foram a óbito. Houve diferença estatística em relação ao desfecho para o uso de ventilação mecânica, noradrenalina, hemodiálise e transfusão de sangue e derivados.

A identificação de os pacientes no uso destes tratamentos durante a internação na UTI terem tido maior mortalidade, logicamente, pode ser atribuída à gravidade da doença. Porém, verificou-se que poucos pacientes obtiveram benefícios quando tais tratamentos foram iniciados após o $7^{\circ}$ dia. Claro está que o número de pacientes receptores deles somente após o $7^{\circ}$ dia não é grande o suficiente para justificar qualquer inferência. No entanto, é possível abrir discussões em torno da questão, na medida em que se verificou ter havido aumento da mortalidade entre os pacientes no uso destes tratamentos durante a internação na UTI e serem eles mais freqüentemente ofertados durante os primeiros sete dias de internação. Propõe-se, então, a partir desses achados, abordar questões sobre futilidade ao relacionar a oferta dos tratamentos em duas fases: fase precoce de intervenção (os primeiros sete dias) e fase tardia de intervenção (após o $7^{\circ} \mathrm{dia}$ ).

Com esse fim, procurou-se comparar, por meio do modelo UNICAMP II, a evolução do risco provável de morte entre pacientes que tiveram alta e pacientes que foram a óbito. Os dados mostraram ser o risco de morte significativamente maior, em todos os tempos, entre os pacientes que foram a óbito. Interessante foi notar que, para os sobreviventes, em média, o risco provável de morte ter ido decrescendo na medida do aumento do tempo, em dias. No entanto, para os pacientes que morreram, o risco provável de morte manteve-se nos mesmos patamares iniciais. Isto sugere que, para pacientes com resposta positiva ao tratamento empregado na fase precoce, o risco de morte diminuirá de forma progressiva e, conseqüentemente, haverá maior probabilidade de alta. Porém, para os que, apesar dos tratamentos empregados, o risco de óbito se mantiver nos mesmos patamares iniciais ou mesmo aumentar, suas chances de sobrevivência serão menores.

Verificou-se também que, entre os 150 pacientes, 17 apresentaram risco provável de morte maior ou igual a $90 \%$ durante os primeiros sete dias de internação na UTI. A sensibilidade a partir de uma única medida foi de $23 \%$, a especificidade de $97 \%$ e o valor preditivo positivo de $82,4 \%$. Constatou-se, então, que, apesar da alta especificidade, basear decisões sobre futilidade terapêutica em uma única medida também não seria plausível pois não refletiria a evolução do quadro clínico do paciente frente ao emprego dos tratamentos mantenedores da vida. Constatou-se, também, a existência de forte correlação $(r>0,83)$ entre os dois modelos (APACHE II e UNICAMP II) no que se refere à intensidade e à direção da associação linear em todos os tempos de aferição. Então, estimou-se o risco de morte individual $\pi$, baseados em ambos os modelos e verificou-se, por meio de ajustes estatísticos correspondentes aos valores de Deviance e AIC, que a qualidade dos dois modelos é estatisticamente significativa, demonstrando, assim, serem os dois métodos equiparáveis.

Sob o ponto de vista dos autores, o modelo UNICAMP II apresenta vantagens visto não utilizar categoria diagnóstica, evitando a dificuldade e o possível engano no enquadramento da categoria correspondente, bem como ser de mais fácil aplicabilidade à beira do leito. Além disso, o modelo UNICAMP II reflete a realidade brasileira, pois foi elaborado a partir de banco de dados coletados no Brasil enquanto o modelo APACHE II, representa a realidade de bancos de dados coletados nos Estados Unidos e Europa onde, certamente existem diferenças, em relação ao Brasil, não somente em nível de atendimento pré-hospitalar, mas também no estado de saúde da população, nível de nutrição e a conseqüente resposta imunológica às doenças. Dados do estudo IBRANUTRI (Inquérito Brasileiro de Avaliação Nutricional) ${ }^{10}$ demonstram que, aproximadamente, $48,1 \%$ da população hospitalizada apresenta algum grau de desnutrição e $12,6 \%$ são desnutridos graves. Os resultados deste estudo são semelhantes aos de Alves e col. ${ }^{11}$ que aplicaram o modelo UNICAMP II para estimar o risco provável de óbito em uma UTI privada 
na cidade de Cuiabá (MT) e o compararam ao modelo APACHE II. Eles concluíram que o modelo UNICAMP ॥ se adapta perfeitamente a um hospital geral não-público e sugeriram extrapolar o novo modelo para a população brasileira, apoiados em dados multicêntricos. Os resultados deste estudo corroboram essas sugestões por ter sido desenvolvido em um hospital universitário de referência nacional, o qual contempla tanto pacientes procedentes do sistema público quanto do privado. Nosso estudo também procurou comparar o modelo APACHE II ao modelo UNICAMP II a partir de um protocolo evolutivo de morte individual $\pi$. Verificou-se, por meio de várias combinações de variáveis explicativas, que a melhor combinação de variáveis seria o índice prognóstico estimado no $1^{\circ}$ dia, seguido da variável de evolução prognóstica. A variável de evolução prognóstica envolvendo $\circ 7^{\circ}$ dia foi a que mostrou menores valores de AIC, sendo aceita para ambos os modelos. Constatou-se, mais uma vez, ser o modelo UNICAMP II equiparável aos níveis internacionais de predição prognóstica.

A partir da análise da criação da variável de evolução prognóstica, para os 112 pacientes com 7 ou mais dias na UTI, verificou-se ter sido a evolução dos índices bem mais acentuada para pacientes com alta, em contraste aos que foram a óbito. Isto demonstrou, mais uma vez, que apesar do emprego de tratamentos invasivos e intensivos, em média, os pacientes que evoluíram para o óbito pioraram o seu índice prognóstico de morte individual $\pi$.

A análise dos dados entre a probabilidade de morte individual $\pi$ e o desfecho, bem como em relação aos tratamentos mantenedores da vida, mostrou novamente ter havido diferenças significativas quanto ao desfecho, para ventilação mecânica, para noradrenalina, para hemodiálise, para transfusão de sangue e derivados e agora também para nutrição parenteral. Ainda céticos quanto aos resultados encontrados, em virtude de, em muitos estudos, o número de pacientes terem sido muito superiores, procurou-se, através de recursos estatísticos, calcular e analisar, a partir dos dados reais, um banco de dados virtual primeiramente com 5000 pacientes e, posteriormente, com 10.000 pacientes. Atribuiu-se níveis de evolução prognóstica de $-20 \%,-10 \%$ e $+10 \%$, ou seja, quanto mais negativa fosse a evolução, melhor seria o prognóstico e melhores as probabilidades de alta. Os dados mostraram, ao analisar a curva de características operacionais (Curva-ROC) que, quando um paciente apresentar risco provável de óbito inicial em torno de 70 a $80 \%$ e, ao longo dos sete primeiros dias, apesar dos tratamentos empregados, piorar o seu prognóstico em $10 \%$, pelo sistema UNICAMP II, a sensibilidade do teste, respectivamente, é de $23,5 \%$ e $16,3 \%$ e a especificidade é, respectivamente, de $97,4 \%$ e $98,6 \%$.

A partir desses resultados, com a obtenção de uma alta especificidade, apesar da baixa sensibilidade, parece-nos razoável levantar a discussão sobre futilidade terapêutica. Em cada 100 pacientes que não apresentarem essa evolução, em torno 98 a 99 pacientes sobreviverão ao tratamento na UTI. No entanto, para aqueles que a apresentarem, somente um a dois pacientes sobreviverão a essa internação. Assim demonstra-se, sistematicamente, não ser possível promover a recuperação do quadro clínico do paciente em níveis aceitáveis que acarretem o benefício de sua alta para os leitos de internação hospitalar. Quando isso não é sistematicamente possível, pode-se caracterizar como futilidade terapêutica.

\section{CONCLUSÃO}

Foi observado neste estudo que o modelo de predição prognóstica UNICAMP II é factível, e poderia ser utilizado nas UTI brasileiras, pois é equiparável aos níveis de modelos internacionais como o APACHE II, de mais fácil aplicabilidade à beira do leito, não necessitando do enquadramento em categoria diagnóstica. Também se verificou a não-existência de critérios claros para sugerir o impedimento do uso efetivo dos tratamentos mantenedores da vida com bases no diagnóstico de uma doença crônica ou aguda grave, raça, idade ou tempo de utilização dessas terapias. Constatou-se ainda, que a fase de intervenção precoce (primeiros 7 dias), é de grande auxílio do ponto de vista objetivo na tomada de decisões éticas em torno da não-oferta de tratamentos mantenedores da vida. Assim, sugerese a não-oferta de mais tratamentos referentes tanto à intensidade quanto à quantidade para pacientes cuja evolução, nos primeiros setes dias de internação, se mostrar desfavorável. Isso seria verificado para aqueles cujo índice prognóstico inicial, de probabilidade de morte, fosse igual ou maior que $70 \%$ e, apesar dos tratamentos empregados, piorarem seu índice prognóstico em $10 \%$ ao longo dos primeiros sete dias de internação na UTI. Nesse sentido, estar-se-ia contemplando o princípio da não-maleficência ao nos reportarmos ao conceito de não tentar, obstinadamente, prolongar a quantidade de vida sem nenhum benefício razoável. Os resultados deste estudo, certamente necessitarão 
BATISTA, GOLDBAUM JÚNIOR, SZTILER E COL.

ser confirmados por outros estudos maiores e multicêntricos em nosso país e/ou no exterior.

\section{REFERÊNCIAS}

01. Workman S, Mckeever P, Harvey $\mathrm{W}$ et al - Intensive care nurses and physicians experiences with demands for treatment: some implications for clinical practice. J Crit Care, 2003;18:17-21.

02. Watts CM, Knaus WA - The case for using objective scoring systems to predict intensive care unit outcome. Crit Care Clin, 1994;10:73-92.

03. Hyzy RC - ICU scoring and clinical decision making. Chest, 1995;107:1482-1483.

04. Terzi RG, Gomez MI, Araujo S et al - Índices prognósticos em Medicina Intensiva. RBTI, 2002;14:6-20.
05. Knaus WA, Draper EA, Wagner DP et al - APACHE II: a severity of disease classification system. Crit Care Med, 1985;13:818-829.

06. Dobson AJ - An Introduction to Generalized Linear Models. $2^{\text {nd }}$ Ed, New York, Chapman \& Hall/CRC, 2002.

07. Jekel FJ, Elmore JG, Katze DL - Epidemiologia, Bioestatística e Medicina Preventiva. $1^{\text {a }}$ Ed, Porto Alegre, ArtMed; 1999.

08. Esserman L, Belkora J, Lenert L - Potentially ineffective care. A new outcome to assess the limits of critical care. JAMA, 1995;274:1544-1551.

09. Joynt GM, Gomersall CD, Tan P et al - Prospective evaluation of patients refused admission to an intensive care unit: triage, futility and outcome. Intensive Care Med, 2001;27:1459-1465.

10. Waitzberg DL, Caiaffa WT, Correia MI - Hospital malnutrition: the Brazilian national survey (IBRANUTRI) a study of 4000 patients. Nutrition, 2001;17:573-580.

11. Alves CJ, Terzi RGG, Franco GPP et al - Comparação entre o modelo UNICAMP II e o APACHE II em uma UTI geral. RBTI, 2003;15:144-152. 